\title{
Upregulation of IncRNA DQ679794 contributes to preventing the progression of hepatocellular carcinoma both in vitro and in vivo
}

\section{Ze-wei Lin}

Peking University Shenzhen Hospital

\section{Qing-qi Ren}

Peking University Shenzhen Hospital

\section{Zhi-feng Huang}

Peking University Shenzhen Hospital

Ji-kui Liu (D w1351003@126.com )

Peking University Shenzhen hospital

\section{Research}

Keywords: IncRNA DQ679794, Hepatocellular carcinoma, Therapeutic target

Posted Date: February 6th, 2020

DOI: https://doi.org/10.21203/rs.2.22787/v1

License: (c) (i) This work is licensed under a Creative Commons Attribution 4.0 International License. Read Full License 


\section{Abstract}

\section{Background}

Hepatocellular carcinoma (HCC) is an aggressive tumor with a poor prognosis. Long noncoding RNAs (IncRNAs) are key regulators of tumor development. However, IncRNA profiles in HCC remain largely unknown. In previous studies, we found that IncRNA DQ786243 plays an important role in the pathogenesis of HCC and promotes the development of HCC. In this study, we investigated the role of IncRNA DQ679794 in the pathogenesis of HCC.

Methods and Results

We first used quantitative real-time PCR among 64 paired HCC tissues, and the level of IncRNA DQ679794 was found to be significantly lower in tumors than in normal tissues. In addition, the effects of IncRNA DQ679794 were assessed by overexpression in vitro and in vivo. We found that the level of apoptosis was increased and that cell proliferation was weakened in HepG2 cells overexpressing DQ679794. Finally, the transplanted tumor experiment confirmed that after the overexpression of IncRNA DQ679794, the growth of transplanted tumors formed by liver cancer cells was inhibited.

\section{Conclusion}

This study suggests that IncRNA DQ679794 is an oncogene that inhibits tumor progression, and we believe that IncRNAs may be a key regulatory center in HCC progression.

\section{Introduction}

Hepatocellular carcinoma (HCC) is one of the most common and malignant tumors in the world and causes approximately 600,000 deaths each year [1]. HCC is the sixth most common cancer worldwide and the third leading cause of cancer-related death [2]. Chronic hepatitis $\mathrm{B} / \mathrm{C}$ virus infections are significant development factors of HCC [3]. Frequent intrahepatic and extrahepatic metastases lead to low respectability, poor prognosis and high recurrence rate in the early stage [4]. Therefore, it is important to find effective methods for the early diagnosis and treatment of HCC.

Long noncoding RNAs (IncRNAs), a novel class of noncoding RNAs, are commonly defined as RNA molecules longer than 200 nucleotides in length [5]. Unlike smaller noncoding miRNAs, the functions of most IncRNAs are not fully understood. However, with the continuous improvement of transcriptome spectroscopy techniques, emerging research has indicated that IncRNAs play a vital role in human tumorigenesis and progression by serving as tumor oncogenes or suppressors [6, 7]. Abnormal expression of IncRNAs has been found in a variety of tumors, including HCC, and is associated with the proliferation, growth, apoptosis, invasion and metastasis of tumor cells $[8,9]$. IncRNAs that have been widely reported in HCC include MALAT1, H19, HULC and MEG3. For example, the levels of MALAT1 and $\mathrm{H} 19$ in normal liver cells were very low, and their expression significantly increased when liver cancer 
occurred[10]. The expression of $\mathrm{H} 19$ in most primary liver cancers is higher than alpha-fetoprotein, and its combined detection with alpha-fetoprotein can improve the diagnosis rate of early liver cancer [11]. HULC can be used as a marker for liver metastasis of colorectal tumors [12]. CUDR can inhibit cysteine aspartic proteinase 3-induced drug resistance [13]. Overexpression of MEG3 could inhibit the growth of HCC cells by inducing the apoptosis of HCC cells $[14,15]$.

However, most of the IncRNA profiles in HCC remain largely unknown. In previous studies, we found that IncRNA DQ786243 plays an important role in the pathogenesis of HCC and promotes the development of HCC [16]. In the present study, we focused on IncRNA DQ679794 to identify the key IncRNA associated

with HCC and to study its biological functions in HCC progression. Overexpression of DQ679794 inhibited the proliferation of HCC cells, suggesting its potential utility as a prognostic marker or a therapeutic target.

\section{Methods}

\section{Patients and tissue specimens}

The present study was approved by the Ethics Committee of Peking University Shenzhen Hospital (Shenzhen, China) (Permit Number: BJ-EC-ZN-01.0-A02). Written informed consent was obtained from all patients prior to enrollment in the present study. Paired tumor tissue and adjacent healthy tissue samples were collected from 64 patients diagnosed with HCC between October 2015 and May 2018 at Peking University Shenzhen Hospital and Sun Yatsen Memorial Hospital (Guangzhou, China). None of the patients enrolled in the study had received chemotherapy or radiotherapy prior to surgery. Written consent approving the use of their samples for research purposes was obtained from each patient.

\section{Cell culture}

The HCC cell lines (HepG2, Hep3B) and normal cell line (L02) were purchased from the Cell Resource Center of the Institute of Basic Medical Sciences of the Chinese Academy of Medical Sciences (Beijing, China). The cells were maintained in Dulbecco's modified Eagle's medium (DMEM) supplemented with $10 \%$ fetal bovine serum (FBS; Gibco, Grand Island, NY), $100 \mathrm{U} / \mathrm{ml}$ penicillin and $100 \mathrm{mg} / \mathrm{ml}$ streptomycin. All cell lines were maintained at $37^{\circ} \mathrm{C}$ in a humidified atmosphere with $5 \% \mathrm{CO}_{2}$.

\section{Plasmid construction and transfection}

Transient transfection was performed, and the manufacturer's instructions were followed regarding the use of Lipofectamine reagent (Invitrogen, Carlsbad, CA, USA). Typically, HepG2 cells at $5 \times 10^{6}$ cells/well in 6-well dishes were transfected with $0.5 \mu \mathrm{g}$ of plasmids. Overexpressed DQ786243 transfection plasmid was purchased from SLNco (Shanghai, China). The plasmids were grown in bacteria following standard techniques and purified with a Qiagen Plasmid kit (Qiagen, Hilden, Germany). The control group was transfected with the empty vector pLentiLox 3.7 (SLNco, Shanghai, China) following the same instructions for transient transfection. Cells transfected with the pcDNA3.4-DQ679794 expression 
plasmid were used for apoptosis analysis. Cells were harvested after $48 \mathrm{~h}$ of transfection and for CCK-8 assays after Day 1, Day 2, Day 3, Day 4, and Day 5 of transfection.

\section{RNA extraction and quantitative real-time PCR}

Tissue samples and serum RNA were extracted with TRIzol reagent and TRIzol LS reagent (Invitrogen, Carlsbad, CA), according to the manufacturer's protocol. Total RNA was reverse transcribed using the PrimeScript RT Reagent Kit with gDNA Eraser (Takara, Dalian, China). Quantitative real-time PCR (qRTPCR) was performed in the LightCycler 480 System using the SYBR Premix ExTaq Kit (Takara). The primers used were as follows: IncDQ DQ679794 forward, 5'-ACCCTCCTCTCCGTCCATAC-3' and reverse, 5'CACCTGGCAGTTGAGTCAGA-3'; GAPDH forward, 5'-AGCCAC ATCGCTCAGACAC-3' and reverse, 5'GCCCAATACGACCAAATCC-3'. The relative expression levels were calculated by the $2^{-}{ }^{\Delta} \mathrm{CT}$ method. Each assay was performed in triplicate.

\section{Western blot}

Transfected cells were dissolved using radioimmunoprecipitation assay (RIPA) buffer supplied with a protease inhibitor cocktail (Sigma-Aldrich, St. Louis, MO, USA). Briefly, the total protein was separated by $10 \%$ SDS-PAGE and then immediately transferred onto PVDF membranes (GE Healthcare, USA). The membranes were blocked using nonfat milk and were then incubated at $4{ }^{\circ} \mathrm{C}$ overnight with primary antibodies against Caspase-3, Caspase-9, Bax, Bcl-2, Cyclin-B1, CDC2 (1:1000; Abcam) and GAPDH (1:2000; Abcam) according to the manufacturer's instructions. Subsequently, the membranes were incubated with secondary antibodies properly at room temperature. The experimental results were visualized with ECL detection reagents (Boster Biological Technology, Pleasanton, CA).

\section{Flow cytometry analysis}

Transfected HepG2 cells were kept in 6-well plates $\left(1 \times 10^{6}\right.$ cells/well) for $48 \mathrm{~h}$ before being rinsed with PBS. Then, the rinsed cells were stained using the FITC Annexin V Dead Cell Kit (BD Biosciences, USA) according to the manufacturer's protocol. The cell apoptosis rate was evaluated with a FACSCalibur flow cytometer (BD Biosciences, Franklin Lakes, NJ, USA).

\section{In vivo xenograft experiments}

BALB/c nude mice aged 6-7 weeks and weighing 20-22 g were used in the experiment. The animal study was performed at Peking University Shenzhen Hospital with approval from the Institutional Animal Care and Use Committee in accordance with the institutional guidelines (). BALB/c nude mice were administered approximately $1 \times 10^{7}$ cells in $200 \mu \mathrm{L}$ of medium. Each experimental group consisted of 811 mice. After 30 days, the mice were killed, and their tumors were excised. The tumor weight was measured, and the tumor volume was calculated according to the formula: tumor volume $\left(\mathrm{mm}^{3}\right)=$ $\left(w^{2}\right) / 2$, where $w$ is the longest axis $(\mathrm{mm})$ and $h$ is the shortest axis $(\mathrm{mm})$.

\section{Data analysis}


Data were analyzed using GraphPad Prism software. Statistical analysis was performed using unpaired ttest measures ANOVA followed by the Bonferroni post hoc test, which were specifically stated in the Results. All results are shown as the mean \pm S.E.M. $P<0.05$ was considered statistically significant.

\section{Result}

\section{IncRNA DQ679794 expression in patients with HCC and cell lines}

The expression levels of IncRNA DQ679794 in patients with HCC and paired nontumor tissues were determined using qRT-PCR. The results showed that IncRNA DQ679794 expression was significantly lower in HCC tumors than in corresponding nontumors (Fig. 1A, ${ }^{\star *} \mathrm{p}<0.01$ ). Moreover, we detected several different HCC cell lines, and the results showed that the level of IncRNA DQ679794 in the HepG2 group and Hep3B group was significantly lower than that in the L02 group (Fig. 1B, ${ }^{\star} \mathrm{p}<0.01$ ). These data suggested that abnormal IncRNA DQ679794 expression may be related to HCC progression.

\section{Effect of IncRNA DQ679794 on HCC cell proliferation and apoptosis in vitro}

As the level of IncRNA DQ679794 is significantly downregulated in the tumor tissues of HCC and HCC cell lines, it may function as a tumor oncogene. To confirm the functional role of IncRNA DQ679794 in HCC, we employed a plasmid to specifically overexpress IncRNA DQ679794 in HepG2 cells (Fig. 2A, **p< 0.01). We first examined the effect of overexpression of IncRNA DQ679794 (DQ-OE group) on the proliferation of HepG2 cells. The CCK-8 assay showed that the DQ-OE group notably retarded cell proliferation in HepG2 cells (Fig. 2B, ${ }^{\star \star} \mathrm{p}<0.01$ ).

In addition, we determined whether apoptosis contributes to cell growth inhibition by IncRNA DQ679794 overexpression. We detected the effect of IncRNA DQ679794 on apoptosis by flow cytometry, and the results showed that the apoptosis level of the DQ-OE group increased significantly compared to that of the DQ-veh group (Fig. 2C, 2D, * $p<0.05$ ). These results indicate that the overexpression of DQ679794 may promote apoptosis in HCC cells.

Moreover, we examined the expression of certain proteins involved in apoptosis regulation by western blot. A significant elevation in proapoptotic protein (Caspase-3 and Caspase-9) levels was observed in the DQ-OE group, and the level of Bax was unchanged compared to the DQ-veh group (Fig. $2 E, 2 F,{ }^{*} p<0.05$, $\left.{ }^{*} \mathrm{p}<0.01\right)$. The expression of the antiapoptotic protein $(\mathrm{Bcl}-2)$ was significantly decreased in the DQ-OE group compared to that in the DQ-veh group (Fig. 2E, $2 \mathrm{~F}$, ${ }^{*} \mathrm{p}<0.01$ ). These results suggest that the dysregulation of certain cell apoptosis-related proteins explains the IncRNA DQ679794-dependent effects on HCC cell proliferation.

\section{IncRNA DQ679794 is involved in cell cycle progression}


Since the induction of cell cycle arrest is an important antiproliferation mechanism, we investigated whether the growth inhibitory activity of IncRNA DQ679794 overexpression was involved in the control of cell cycle progression. To detect the expression of intracellular proteins that regulate cell cycle progression in the G2/M-phase, we measured the protein levels of Cyclin B1, CDC2 and p-CDC2 (Tyr-15). Our results showed that the level of Cyclin B1 in the DQ-OE group was decreased dramatically, and the level of CDC2 was also significantly decreased compared to that in the DQ-veh group (Fig. 3A, 3B, **p < 0.01). The levels of the inhibitory p-CDC2 (phosphorylated at Tyr-15) in the DQ-OE group were increased dramatically compared to those in the DQ-veh group (Fig. 3A, 3B, ${ }^{*}<0.05$ ). These results suggest that the dysregulation of certain cell cycle-related proteins explains the IncRNA DQ679794-dependent effects on HCC cell proliferation.

\section{Overexpression of IncRNA DQ679794 suppressed the tumorigenicity of HCC cells in vivo}

To further investigate the role of IncRNA DQ679794 in the progression of HCC in vivo and to determine the therapeutic potential of targeting IncRNA DQ679794 in HCC, we established xenograft tumor models using Hep3B and HepG2 cells, which were stably transfected with targeting IncRNA DQ679794 or empty vector and injected subcutaneously into the right dorsal tissues of nude mice. After 30 days, the tumors were harvested as shown in Fig. 4A. We measured the size and weight of the tumor tissue, and the results showed that the tumor size in the overexpressed IncRNA DQ679794 (HepG2-DQ) group was significantly lower than that of the HepG2-veh group (Fig. 4B, ${ }^{\star \star} \mathrm{p}<0.01$ ). In addition, the weight of tumors in the HepG2-DQ group was also significantly lower than that in the HepG2-veh group (Fig. 4C, $* \star p<0.01$ ). These results suggest that upregulating IncRNA DQ679794 inhibits tumor growth in vivo.

\section{Discussion}

$\mathrm{HCC}$ is a leading cause of cancer-related death worldwide, and the recurrence and metastasis of tumors are the main factors leading to poor prognosis of patients with HCC [17]. As a new class of ncRNAs, thousands of IncRNAs have been found to be important regulators of human gene expression. Although most IncRNAs have been functionally described, the vast majority of these RNAs have not been fully described [18]. In addition, recent studies have greatly improved our understanding of the important role of IncRNAs in HCC. In this study, we found differences in IncRNA DQ679794 between HCC tissues and adjacent normal tissues. Our functional test results showed that DQ679794 could effectively inhibit cell proliferation both in vivo and in vitro, suggesting that it plays an important role in inhibiting HCC proliferation.

In the past decade, there have been many reports on IncRNAs and HCC. For example, Wang et al [19]. demonstrated that the expression levels of IncRNA HOXA-AS2 were significantly upregulated in HCC tissues and 4 cell lines. In addition, knocking down HOXA-AS2 significantly inhibited proliferation, colony formation, migration and invasion of HCC cells and obviously induced cancer cell apoptosis [20]. Xiao et al [21]. identified that IncRNA Ftx was upregulated in human HCC tissues and cell lines, especially in 
relation to invasive clinicopathological features. Overexpression of IncRNA Ftx promoted the proliferation, invasion and migration of HCC cells, while knockdown of IncRNA Ftx had the opposite effect [22, 23]. In our previous studies, we also found that IncRNA DQ786243 was elevated in both HCC tissue samples and serum, which was associated with a low survival rate and poor clinicopathological characteristics [16]. In addition, multivariate analysis showed that IncRNA DQ786243 expression was an independent prognostic factor for HCC. Knockdown of DQ786243 induces inhibition of cell proliferation, migration, and invasion in vivo and in vitro [21].

In contrast to our study, the above IncRNA levels were highly expressed in HCC tissues, while the DQ679794 we studied in this paper showed a low expression level in HCC tissues. This suggests that the deletion of DQ679794 may be an important factor affecting the accelerated HCC process. There are also many IncRNAs with low expression in HCC tissues, such as uc.134 and RGMB-AS1 [24]. Wen et al [25]. indicated that the expression of a novel IncRNA uc.134 was repressed in HCC specimens and that the expression of uc.134 was significantly correlated with the overall survival of patients. Moreover, the overexpression of uc.134 suppressed HCC cell proliferation, invasion, and metastasis in vitro and in vivo [26]. Nan et al [27]. demonstrated that IncRNA RGMB-AS1 expression was decreased in hepatocellular carcinoma tissues and cell lines, and low IncRNA RGMB-AS1 expression correlated with malignant status and poor prognosis of patients with hepatocellular carcinoma. Overexpression of IncRNA RGMB-AS1 obviously suppressed hepatocellular carcinoma cell proliferation, migration, and invasion and promoted cell apoptosis [28]. This suggests that IncRNA DQ679794 is a general target for antitumor therapy.

Moreover, to investigate the possible mechanism responsible for the proliferation enhancement effect of DQ679794, we detected proteins associated with the cell cycle and found that overexpression of DQ794697 can block the cell cycle and promote apoptosis in G2/M phase, suggesting that DQ786243mediated HCC cell proliferation may be associated with regulation of the cell cycle and apoptosis. To further elucidate the regulatory mechanism of DQ794697 in the cell cycle and apoptosis, we analyzed the proteins involved in the cell cycle and apoptosis by western blot. Our results showed that overexpression of DQ794697 significantly reduced the expression of Cyclin B1 and increased the phosphorylation of CDC2. It is widely accepted that the Cyclin B1-CDC2 complex is necessary for the transition from G2 phase to M phase[29]. We also observed that DQ794697 gene overexpression in HepG2 cells decreased the expression of the antiapoptotic protein $\mathrm{Bcl}-2$ and increased the expression of the proapoptotic proteins Caspase-9 and Caspase-3. These results may extend our current understanding of the downstream genes of DQ794697 to include these cell cycle- and apoptosis-related proteins.

Although IncRNAs have been reported to be involved in the progression and development of tumors, the underlying molecular mechanisms are unclear [30]. In this study, we found that the expression of IncRNA DQ794697 was correlated with HCC tumor and cell lines. Of course, as the number of HCC cell lines available is limited, these cultured cell lines cannot represent all subtypes of colorectal tumors, and more systematic studies using clinical HCC samples are required, however, it provides a possible overexpression of IncRNA DQ794697 that may be associated with colorectal neoplasia. 


\section{Conclusion}

In conclusion, this study suggests that IncRNA DQ679794 is an oncogene that inhibits tumor progression, and we believe that IncRNAs may be a key regulatory center in HCC progression.

\section{Declarations}

Ethics Approval and Consent to Participate: All animal experiments and procedures were approved by the Ethics Committee of Shenzhen Hospital of Peking University (Permit Number: BJ-EC-ZN-01.0-A02).

Consent for Publication: The authors showed their consent for publication of the research results.

Availability of data and material: The datasets generated and/or analyzed during the current study is available from the corresponding author on reasonable request.

Competing Interest: The authors have no competing interest to declare.

Author Contributions: Data curation, Ze-wei Lin, Qing-qi Ren and Zhi-feng Huang; Formal analysis, Ze-wei Lin and Qing-qi Ren; Funding acquisition, Ji-kui Liu; Project administration, Ji-kui Liu; Writing - original draft, Ze-wei Lin; Writing - review \& editing, Ji-kui Liu.

Funding: Supported by Sanming Project of Medicine in Shenzhen (No. SZSM201612021), Special Foundation for Science and Technology Development of Guangdong Province (No.2017B090904010), Scientific Research Project of Health and Family Planning Commission of Shenzhen Municipality (No. SZXJ2018086) and Research Foundation of Peking University Shenzhen Hospital (No. JCYJ2018006). This work was financially supported by the Science and Technology Development Fund Project of Shenzhen (JCYJ20150403091443331)

Acknowledgments: We are grateful to Shenzhen Hospital of Peking University for their assistance on technical and experiments of animals.

\section{References}

[1] Patel KD, Vora HH, Trivedi TI, Patel JB, Pandya SJ, Jetly DH, et al. Transcriptome profiling and pathway analysis in squamous cell carcinoma of buccal mucosa. Exp Mol Pathol. 2020:104378.

[2] Shi H, Sun H, Li J, Bai Z, Wu J, Li X, et al. Systematic analysis of IncRNA and microRNA dynamic features reveals diagnostic and prognostic biomarkers of myocardial infarction. Aging (Albany NY). $2020 ; 12$.

[3] Xu J, Lu Y, Liu Q, Xia A, Zhao J, Xu X, et al. Long noncoding RNA GMAN promotes hepatocellular carcinoma progression by interacting with elF4B. Cancer Lett. 2019;473:1-12. 
[4] Zheng Y, Nie P, Xu S. Long noncoding RNA linc00467 plays an oncogenic role in hepatocellular carcinoma by regulating the miR-18a-5p/NEDD9 axis. J Cell Biochem. 2020.

[5] Chen Q, Fu L. Upregulation of long non-coding RNA ROR1-AS1 promotes cell growth and migration in bladder cancer by regulation of miR-504. PLoS One. 2020;15:e0227568.

[6] Gao M, Fu J, Wang Y. The IncRNA FAL1 protects against hypoxia-reoxygenation- induced brain endothelial damages through regulating PAK1. J Bioenerg Biomembr. 2020.

[7] Song M, Zhong A, Yang J, He J, Cheng S, Zeng J, et al. Large-scale analyses identify a cluster of novel long noncoding RNAs as potential competitive endogenous RNAs in progression of hepatocellular carcinoma. Aging (Albany NY). 2019;11:10422-53.

[8] Wang S, Li G. LncRNA XIST inhibits ovarian cancer cell growth and metastasis via regulating miR-1505p/PDCD4 signaling pathway. Naunyn Schmiedebergs Arch Pharmacol. 2020.

[9] Yang X, Xin N, Qu HJ, Wei L, Han Z. Long Non-Coding RNA TUG1 facilitates Cell Ovarian Cancer Progression through Targeting MiR-29b-3p/MDM2 Axis. Anat Rec (Hoboken). 2020.

[10] Lin R, Maeda S, Liu C, Karin M, Edgington TS. A large noncoding RNA is a marker for murine hepatocellular carcinomas and a spectrum of human carcinomas. Oncogene. 2007;26:851-8.

[11] Gabory A, Ripoche M-A, Yoshimizu T, Dandolo L. The $<\mathrm{i}>\mathrm{H} 19</ \mathrm{i}>$ gene: regulation and function of a non-coding RNA.113:188-93.

[12] Matouk IJ, Abbasi I, Hochberg A, Galun E, Dweik H, Akkawi M. Highly upregulated in liver cancer noncoding RNA is overexpressed in hepatic colorectal metastasis. European Journal of Gastroenterology \& Hepatology.21:688-92.

[13] Tsang WP, Wong TWL, Cheung AHH, Co CNN, Kwok TT. Induction of drug resistance and transformation in human cancer cells by the noncoding RNA CUDR. RNA. 2007;13:890-8.

[14] Braconi C, Kogure T, Valeri N, Huang N, Nuovo G, Costinean S, et al. microRNA-29 can regulate expression of the long non-coding RNA gene MEG3 in hepatocellular cancer.30:4750-6.

[15] Braconi C, Huang N, Patel T. MicroRNA-dependent regulation of DNA methyltransferase-1 and tumor suppressor gene expression by interleukin-6 in human malignant cholangiocytes. Hepatology. 2010;51.

[16] Zeng B, Lin Z, Ye H, Cheng D, Zhang G, Zhou J, et al. Upregulation of LncDQ is Associated with Poor Prognosis and Promotes Tumor Progression via Epigenetic Regulation of the EMT Pathway in HCC. Cell Physiol Biochem. 2018;46:1122-33.

[17] Zou RC, Shi ZT, Xiao SF, Ke Y, Tang HR, Wu TG, et al. Co-expression analysis and ceRNA network reveal eight novel potential IncRNA biomarkers in hepatocellular carcinoma. PeerJ. 2019;7:e8101. 
[18] Zeng YL, Guo ZY, Su HZ, Zhong FD, Jiang KQ, Yuan GD. Diagnostic and prognostic value of IncRNA cancer susceptibility candidate 9 in hepatocellular carcinoma. World J Gastroenterol. 2019;25:6902-15.

[19] Wang J, Su Z, Lu S, Fu W, Liu Z, Jiang X, et al. LncRNA HOXA-AS2 and its molecular mechanisms in human cancer. Clin Chim Acta. 2018;485:229-33.

[20] Wang F, Yang H, Deng Z, Su Y, Fang Q, Yin Z. HOX Antisense lincRNA HOXA-AS2 Promotes Tumorigenesis of Hepatocellular Carcinoma. Cell Physiol Biochem. 2016;40:287-96.

[21] Li X, Zhao Q, Qi J, Wang W, Zhang D, Li Z, et al. IncRNA Ftx promotes aerobic glycolysis and tumor progression through the PPARgamma pathway in hepatocellular carcinoma. Int J Oncol. 2018;53:551-66.

[22] Liu F, Yuan JH, Huang JF, Yang F, Wang TT, Ma JZ, et al. Long noncoding RNA FTX inhibits hepatocellular carcinoma proliferation and metastasis by binding MCM2 and miR-374a. Oncogene. 2016;35:5422-34.

[23] Liu Z, Dou C, Yao B, Xu M, Ding L, Wang Y, et al. Ftx non coding RNA-derived miR-545 promotes cell proliferation by targeting RIG-I in hepatocellular carcinoma. Oncotarget. 2016;7:25350-65.

[24] Sheng N, Li Y, Qian R, Li Y. The clinical significance and biological function of IncRNA RGMB-AS1 in hepatocellular carcinoma. Biomed Pharmacother. 2018;98:577-84.

[25] Ni W, Zhang Y, Zhan Z, Ye F, Liang Y, Huang J, et al. A novel IncRNA uc.134 represses hepatocellular carcinoma progression by inhibiting CUL4A-mediated ubiquitination of LATS1. J Hematol Oncol. 2017;10:91.

[26] Li ZB, Han YS, Wei LL, Shi LY, Yi WJ, Chen J, et al. Screening and identification of plasma IncRNAs uc.48+ and NR_105053 as potential novel biomarkers for cured pulmonary tuberculosis. Int J Infect Dis. 2020 .

[27] Sheng N, Li Y, Qian R, Li Y. The clinical significance and biological function of IncRNA RGMB-AS1 in hepatocellular carcinoma. Biomedicine \& Pharmacotherapy.98:577-84.

[28] Ding $\mathrm{CH}$, Yin C, Chen S-J, Wen L-Z, Ding K, Lei S-J, et al. The HNF1a-regulated IncRNA HNF1A-AS1 reverses the malignancy of hepatocellular carcinoma by enhancing the phosphatase activity of SHP-1. Molecular Cancer.17:63.

[29] Gentric G, Maillet V, Paradis V, Couton D, L'Hermitte A, Panasyuk G, et al. Oxidative stress promotes pathologic polyploidization in nonalcoholic fatty liver disease. J Clin Invest. 2015;125:981-92.

[30] Xie G, Huang Z, Liu Z, Lin Z, Ma L. NCPHLDA: a novel method for human IncRNA-disease association prediction based on network consistency projection. Mol Omics. 2019;15:442-50. 
Figures
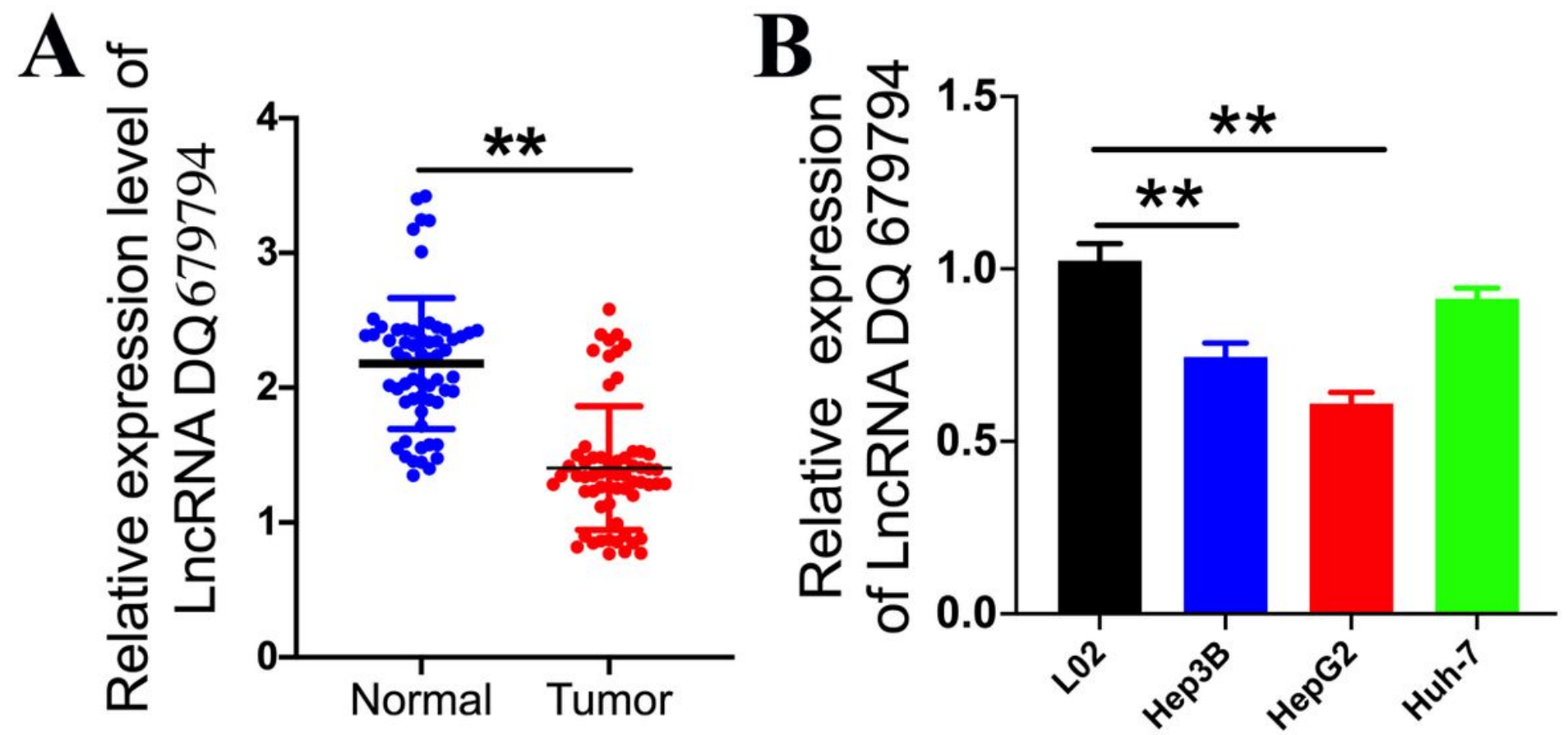

Figure 2

Expression levels of IncRNA DQ679794 in HCC samples and cell lines. A. Relative expression levels of IncRNA DQ679794 in HCC tumor tissues compared with corresponding nontumor tissues $(n=64, * * p<0.01$ versus the normal group). B. Expression levels of IncRNA DQ679794 in HCC cell lines (Hep3B, HepG2, and Huh-7) compared to normal cells (L02) $(n=6, * * p<0.01)$. 


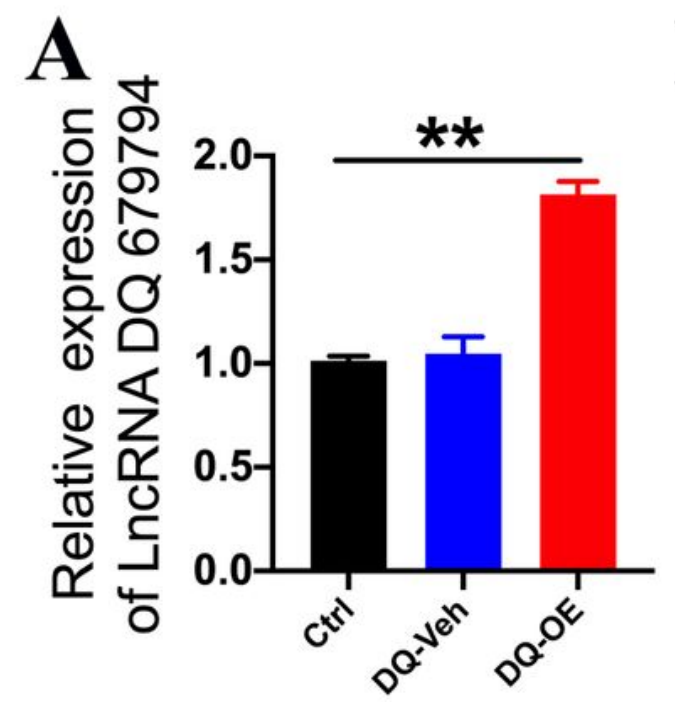

B

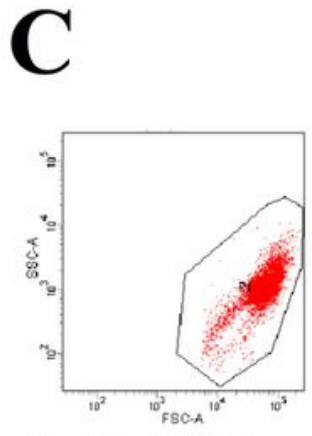

HepG2 Negative

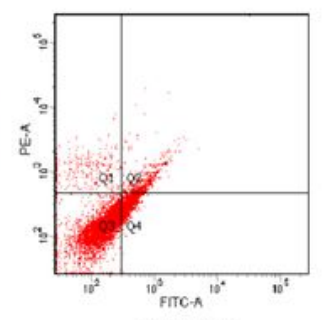

DQ-Veh

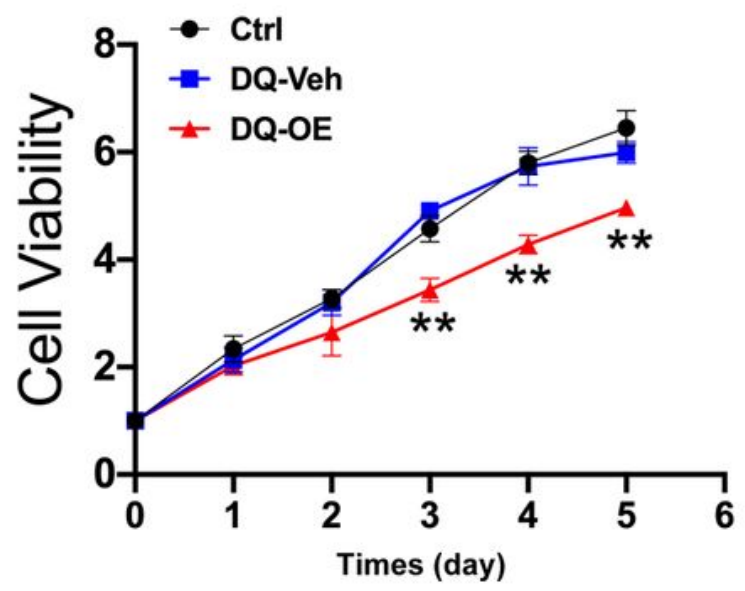

D

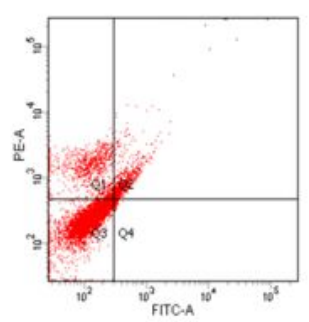

DQ-OE

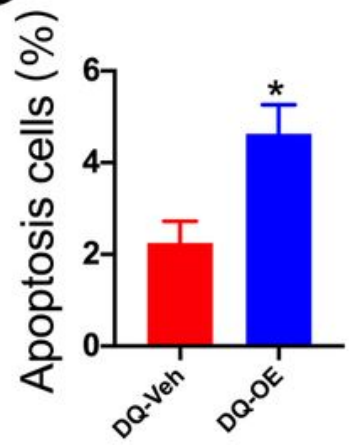

$\mathbf{E}$

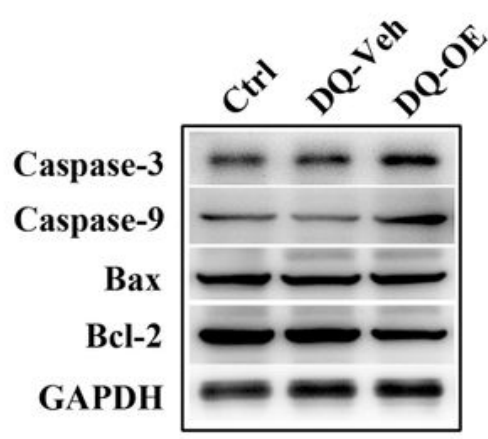

F

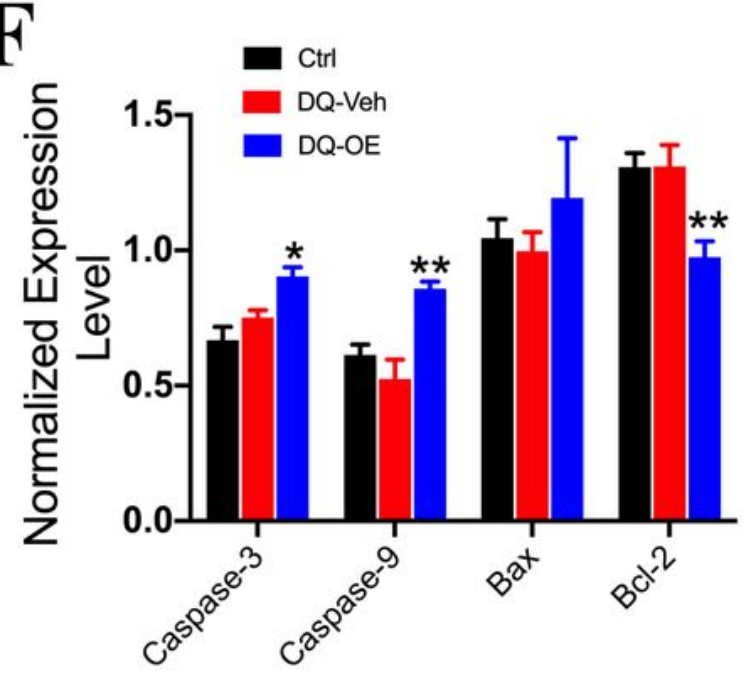

Figure 4

Effect of IncRNA DQ679794 on HCC cell apoptosis in vitro A. Relative expression levels of IncRNA DQ679794 after plasmid transfection (DQ-OE group) compared with the control group (ctrl or DQ-veh group) ( $n=6,{ }^{* \star} p<0.01$ versus the control group). B. CCK-8 cell counts after plasmid transfection and durations (Days 1-5). DQ679794 overexpression notably inhibited cell proliferation in both HepG2 cell lines $(n=6)$. ( ${ }^{\star \star} p<0.01$ versus the control group). C. Apoptosis was assessed using flow cytometry. 
Representative quadrant figures are presented. D. Overexpression of IncRNA DQ679794 greatly increased the number of total apoptotic cells in HepG2 cells $(n=4)$. E. Representative western blot images of the expression of Caspase-3, Caspase-9, Bax, and Bcl-2 in HepG2 cells. F. Densitometric analysis of protein levels in (E). Quantitative results were normalized against the levels of GAPDH. Values are expressed as the mean \pm SEM (error bars) $(n=5)$. ${ }^{\star} p<0.05,{ }^{*} p<0.01$ versus the ctrl group or DQ-veh group.

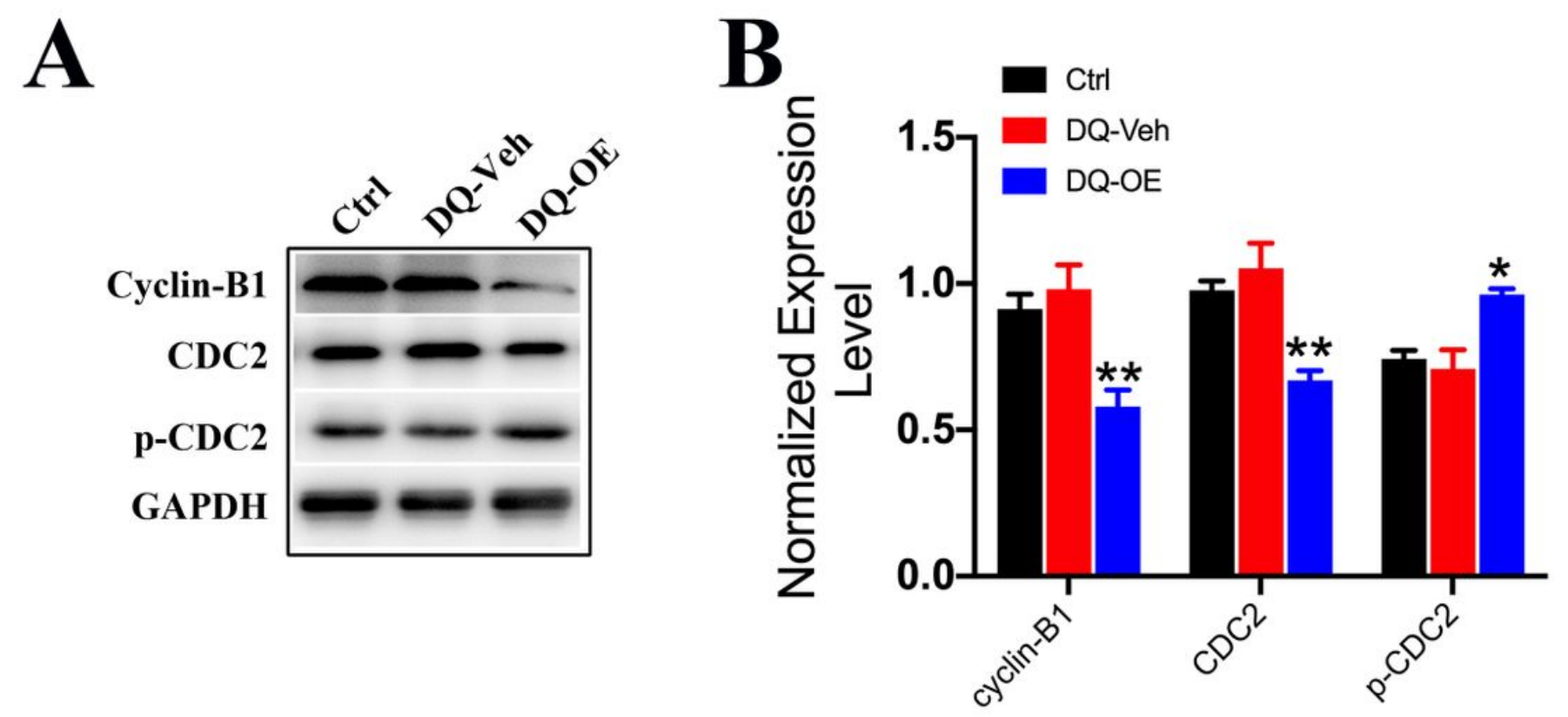

Figure 6

. IncRNA DQ679794 is involved in cell cycle progression. A. Representative western blot images of the expression of Cyclin-B1, CDC2, and p-CDC2 (Tyr-14) in HepG2 cells. B. Densitometric analysis of the protein levels in (A). Quantitative results were normalized against the levels of GAPDH. Values are expressed as the mean \pm SEM (error bars) $(n=5) .{ }^{*} p<0.05$, ${ }^{\star \star} p<0.01$ versus the ctrl group or DQ-veh group. 
A

B
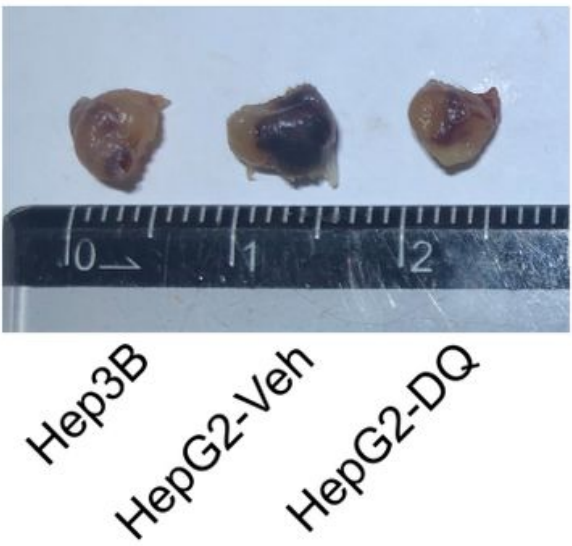

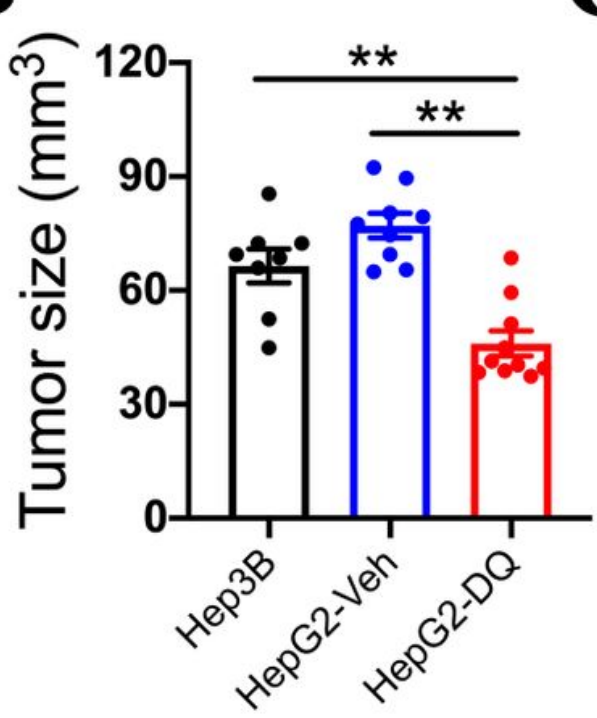

C

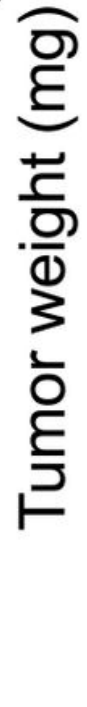

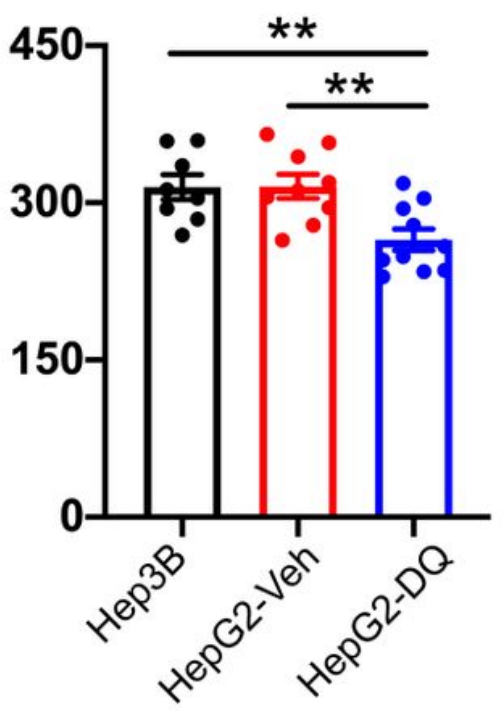

Figure 8

Overexpression of IncRNA DQ679794 suppressed the tumorigenicity of HCC cells in vivo. A. Nude mice were injected with Hep3B and HepG2 cells transfected with DQ679794 (HepG2-DQ group) or vector (HepG2-veh group) via peritoneal injection. After 30 days, the mice were sacrificed, and the tumors were harvested for analysis. B. The tumor volume was measured. C. The tumor weight was measured. Values are expressed as the mean \pm SEM (error bars). $n=8-11,{ }^{*} p<0.05$, ${ }^{\star \star} p<0.01$ versus the Hep3B group or HepG2-veh group. 